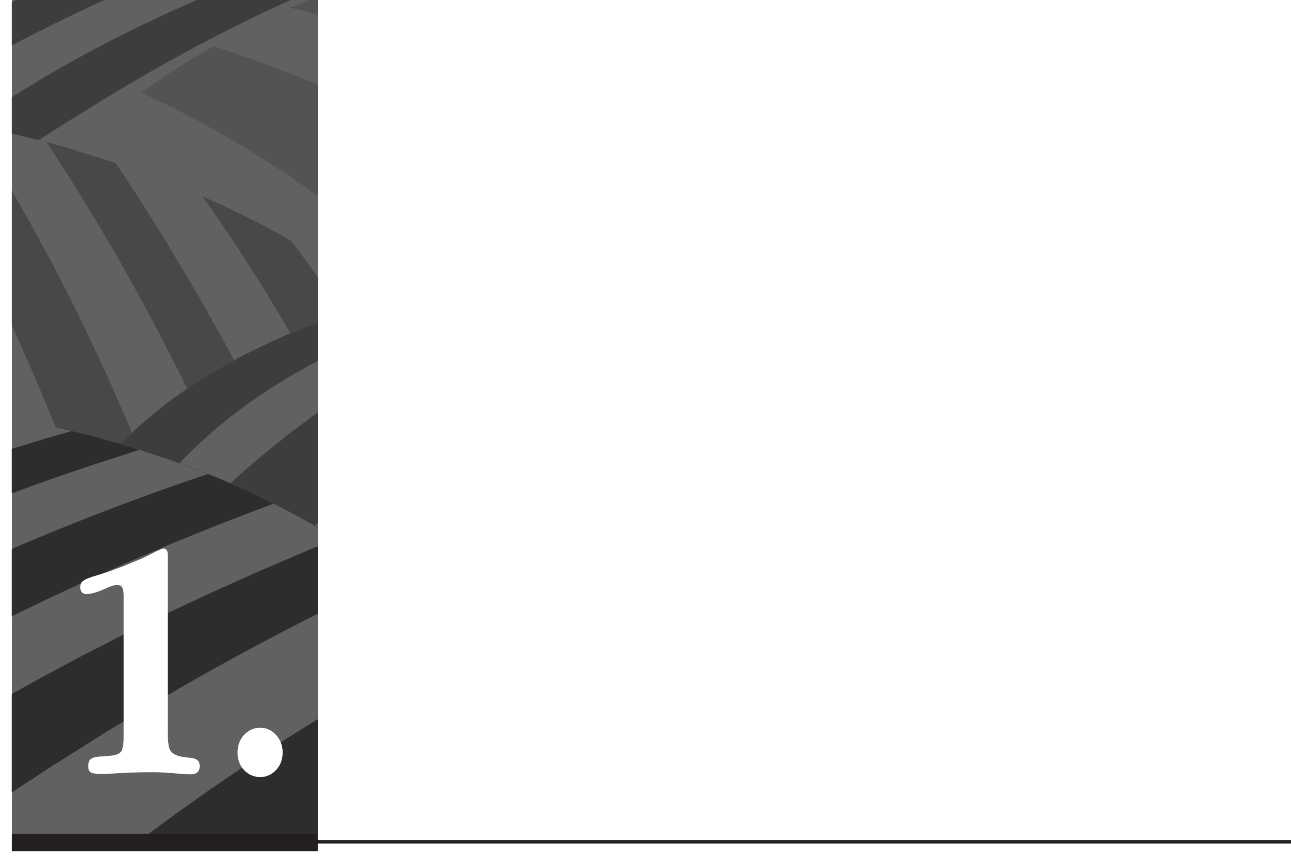

La Responsabilidad Social Empresarial como herramienta para la gestión de conflictos con comunidades rurales 


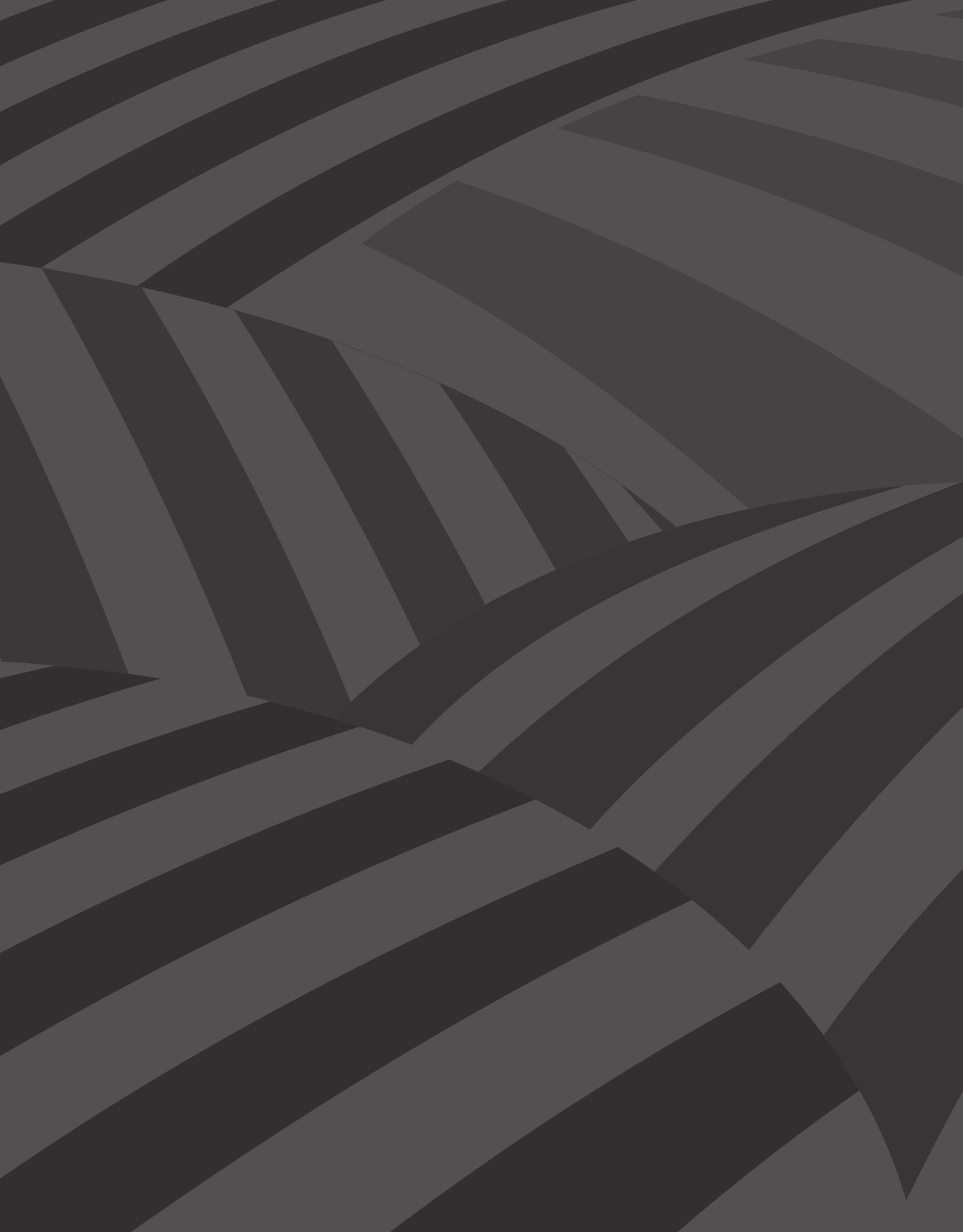




\section{La Responsabilidad Social Empresarial como herramienta para la gestión de conflictos con comunidades rurales}

Por Manuel Ramiro Muñoz*, Daniela Díaz Lozano**, María Alejandra Quintero Falla***

Resumen: Las empresas están llamadas a reflexionar en torno al alcance de sus acciones en el marco de la Responsabilidad Social Empresarial (RSE), más aún, en contextos como Colombia, donde la ruralidad es el escenario de conflictos que involucran a las comunidades indígenas, afrodescendientes y campesinas, lo que supone un reto en la formulación de estrategias de relacionamiento encaminadas a impactar positivamente en la gestión de conflictos que, de no ser tenidos en cuenta por las empresas, pueden ir en detrimento de sus objetivos. Este artículo ofrece una reflexión crítica sobre el concepto de RSE asumida como una herramienta de gestión de conflictos, suponiendo que su alcance debería ir más allá del cumplimiento de las obligaciones legales de la empresa, que debe, entonces, actuar siendo consciente de la asimetría de derechos de las comunidades rurales y de sus consecuencias.

Palabras clave: Responsabilidad Social Empresarial, empresas, comunidades rurales, gestión de conflictos, diálogo intercultural.

* Doctor en Educación de la Universidad de Barcelona, con mención de tesis Excellent Cum Laude. Filósofo, humanista, especialista en Investigación, magíster en Educación. Fundador y director del Instituto de Estudios Interculturales de la Pontificia Universidad Javeriana de Cali. Correo electrónico: mrmunoz@javerianacali.edu.co.

** Antropóloga de la Universidad Icesi. Analista de proyectos en Manglar. Estrategia, Innovación Social y Sostenibilidad. Correo electrónico: dilodany@hotmail.com

*** Politóloga de la Pontificia Universidad Javeriana de Cali y estudiante de la Maestría en Derechos Humanos y Cultura de Paz de la misma universidad. Investigadora de la línea de investigación en Desarrollo Rural y Ordenamiento Territorial del Instituto de Estudios Interculturales de la Pontificia Universidad Javeriana de Cali. Correo electrónico: mquintero98@javerianacali.edu.co. 


\section{Corporate Social Responsibility as a tool for managing conflicts with rural communities}

Abstract: Companies are called to reflect on the scope of their actions in the framework of Corporate Social Responsibility (CSR), even more in contexts such as Colombia, where rurality is the scenario of conflicts involving indigenous, Afro-descendent and peasants communities, this represents a challenge to the formulation of relationship strategies aimed at positively impacting the management of conflicts that, if they are not taken into account by companies, may be detrimental to their objectives. This article offers a critical reflection on the concept of CSR understood as a conflict management tool, assuming that its scope should go beyond the fulfillment of the legal obligations of the company, which must act with awareness of the asymmetry of rights of the rural communities and their consequences.

Keywords: Corporate Social Responsibility, companies, rural communities, conflict management, intercultural dialogue.

Cómo citar este artículo: Ramiro Muñoz, Manuel; Díaz Lozano, Daniela y Quintero Falla, María Alejandra (2019). La Responsabilidad Social Empresarial como herramienta para la gestión de conflictos con comunidades rurales. Revista Controversia, 212, 13-46.

Fecha de recepción: 12 de noviembre de 2018

Fecha de aprobación: 13 de febrero de 2019

\section{Introducción}

$\mathrm{E}$ 1 auge de los conflictos armados intraestatales en países caracterizados por una amplia diversidad cultural, trae consigo un reto significativo en cuanto al papel que el sector privado se encuentra llamado a desempeñar en los procesos de construcción de paz, toda vez que la falta de atención a los conflictos que tienen lugar en su entorno puede devenir en grandes obstáculos para el cumplimiento de los objetivos de la empresa, que incluso, puede llegar a convertirse en un actor que limite o actúe directamente en detrimento de la construcción de paz.

En ese sentido, las empresas están llamadas a reflexionar cada vez más sobre los impactos directos que sus actividades puedan tener sobre 
las poblaciones presentes en sus áreas de influencia, mayormente si estas son, como en el caso colombiano, comunidades rurales con apremiantes condiciones de vulnerabilidad envueltas en diferentes ciclos de violencia. Tal reflexión no obstante, sin pretender una apelación paternalista de la empresa, sino más bien una perspectiva desde la corresponsabilidad que esta guarda con el Estado, está cada vez más avocada a ser integral, de manera que las estrategias que deriven de ella consideren las múltiples dimensiones de los sujetos relacionados de una $\mathrm{u}$ otra forma con el accionar de la empresa, lo que pasa, entonces, por cuestionarse si su labor debería ir —o no- más allá de la minimización de sus impactos negativos para, por el contrario, convertirse en un agente activo con relación a la gestión de conflictos.

Especialmente en Colombia, asumir una perspectiva integral sobre los entornos rurales donde se puedan llegar a desempeñar las actividades del sector privado implica tener en cuenta que hablar de conflictos no debería conducir a pensar únicamente en conflictos armados, pues en países como el nuestro, los diferentes actores sociales no solo resultan víctimas de este, sino que también se ven frecuentemente envueltos en conflictos interétnicos e interculturales —algunas veces atizados por el mismo conflicto armado-, vinculados fuertemente con las asimetrías en el reconocimiento de derechos entre las comunidades étnica y culturalmente diferenciadas, por cuenta del modelo multicultural implementado en el país tras la Constitución Política de 1991. En ese contexto, se hace entonces necesario repensar las herramientas de que dispone la empresa para su contribución a la gestión de conflictos y construcción de paz con un enfoque ciertamente intercultural.

Teniendo en cuenta lo anterior, este artículo tiene por objetivo realizar una reflexión crítica sobre el concepto de Responsabilidad Social Empresarial (en adelante RSE) con enfoque intercultural como una herramienta para la gestión de conflictos en que se vinculen comunidades rurales étnica y culturalmente diferenciadas (indígenas, afrodescendientes y campesinas). Para ello, en primer lugar se desarrolla un 
trabajo teórico conceptual sobre la RSE. En segundo lugar, se hace una reflexión en torno al debate sobre su voluntariedad/obligatoriedad, y en tercer lugar, se lleva a cabo un análisis sobre el posible uso de RSE con perspectiva intercultural como un medio para la gestión de conflictos.

\section{El concepto de Responsabilidad Social Empresarial}

Respecto del concepto de Responsabilidad Social Empresarial o Corporativa (RSE) que guiará la reflexión propuesta, es preciso comenzar por apuntar que, pese a ser abundante la literatura desarrollada sobre el tema, no se trata aquí de realizar una revisión exhaustiva sobre la misma. No obstante, y para efectos de una pertinente, pero breve aproximación histórica a la teoría de la RSE, consideramos útil partir por remontarse a la postura que sobre el tema predominó durante el siglo XVIII y gran parte del XIX, fuertemente marcada por la consolidación de Estados Unidos de América y los procesos de industrialización, según la cual la única responsabilidad reconocida por las empresas debía ser la maximización de utilidades para sus accionistas (Murphy, 1978; Yepes, Peña y Sánchez, 2007), tratándose, entonces, de una responsabilidad exclusivamente económica y con propósitos utilitaristas.

Una primera referencia sobre el concepto (más no sobre el término) de responsabilidad social llegaría en 1889 con Andrew Carnegie en su libro The Gospel of Wealth, en el que planteaba que «las personas adineradas y sus empresas deberían asumir como responsabilidad propia el administrar la riqueza de sus organizaciones para así luego brindar beneficios a la sociedad y socorrer a los menesterosos» (Yepes et al., 2007). Como se advierte, el surgimiento del concepto denota un sentido filantrópico de ayuda a los más necesitados, a través de la administración de los excedentes de capital. Este periodo (bautizado por Murphy como la «fase filantrópica»), en el que se ubican los planteamientos de Carnegie, se extendería desde 1900 hasta 1950. 
En ese orden de ideas, la primera conceptualización de la empresa como actor envuelto en los asuntos sociales y morales se caracterizó por ser de corte filantrópico y paternalista. Esta postura de «ayuda» voluntaria y desinteresada por parte de las grandes compañías se fundamentó en varias motivaciones, entre las que Yepes et al. (2007) destacan: la caridad, la mercantilización de las inversiones sociales para lograr impacto económico y publicitario y los beneficios tributarios. Por su parte, Duque, Cardona y Rendón (2013) exponen cómo la Gran Depresión crisis económica que se extendió en la década de 1930- y la Segunda Guerra Mundial fraguaron un clima de extrema pobreza y escasez que dotó de sentido a la RSE en esta versión filantrópica.

Ahora bien, los estudios sobre responsabilidad social, propiamente dichos, se inauguraron en la década de 1950 con el libro Social Responsibilities of the Businessman (1953) de Howard Bowen. Llamado «el padre de la Responsabilidad Social Corporativa» (Carroll, 1999, p. 270), Bowen planteó que las acciones y las decisiones implementadas por los empresarios tienen un impacto directo en el resto de la sociedad, así definió la responsabilidad social de las empresas como «las obligaciones de los empresarios para aplicar políticas, tomar decisiones o seguir las líneas de acción que son deseables en términos de los objetivos y valores de nuestra sociedad» (Bowen, como se citó en Carroll, 1999, p. 270). Su postura rescata, entonces, el servicio a la sociedad y los fines sociales de las corporaciones, antes que sus funciones económicas. La particularidad de su perspectiva se hallaría en el paso «de un principio de caridad (acciones filantrópicas lideradas por empresarios sensibles) a un principio de administración, en el que las acciones sociales son integradas en la estrategia de empresa» (Navarro, 2013, p. 60).

Una vez abierto el debate académico y social sobre el tema, las proclamas de cambio de la década de 1960 contribuyeron a complejizarlo. Yepes et al. (2007) señalan que, a las consideraciones sobre competitividad, se sumó la presión generada por las acciones y exigencias sindicales y el embrionario posicionamiento de la conservación medioambiental en 
el escenario global, razón por la que en este periodo surgieron perspectivas diversas sobre el concepto de RSE, entendiéndose bien como una postura pública sobre cómo usar los recursos para el bienestar socioeconómico de la sociedad, como obligaciones corporativas que superan lo económico-jurídico o incluso, como las condiciones dadas para el uso responsable del poder empresarial (Frederick; McGuire y Davis, como se citaron en Carroll, 1999).

Sin embargo, el reconocimiento de la RSE como asunto a incluir en las prácticas corporativas no surgió sin detractores. Entre sus críticos acérrimos se destaca Milton Friedman, exponente de las teorías instrumentales de la «responsabilidad social corporativa», en términos de Garriga y Melé (2004). Siguiendo a estos autores, las teorías instrumentales son aquellas que conciben a la empresa únicamente como un medio para generar utilidades, por lo cual las acciones de RSE representan una herramienta estratégica para alcanzar los objetivos económicos trazados por la compañía. Friedman, en su libro Capitalismo y libertad (1962), arguyó que la única responsabilidad de los negocios frente a la sociedad es incrementar sus ganancias, dentro de las reglas legales y las costumbres éticas vigentes, postura que parte del liberalismo para defender el libre mercado y señalar al sector público (y no al privado) como el debido «encargado de proteger (...) los derechos de los trabajadores y de los consumidores, la práctica de los derechos humanos y la protección del medio ambiente» (Yepes et al., 2007, p. 100), doctrina que no es ajena a la que aún hoy algunas empresas defienden bajo el argumento de que las acciones socialmente responsables corresponden al Estado y no a ellas.

A pesar de las críticas, la exploración sobre la RSE continuó su curso. Entre las décadas de 1960 y 1970 las luchas sociales y políticas impactaron profundamente las elaboraciones teóricas que se hicieron al respecto, en tanto cuestionaron sistemáticamente el accionar corporativo en términos sociales, éticos, ambientales e incluso en lo referente al 
modelo económico predominante, promoviendo con ello el debate en torno al papel de la ética en los contextos organizacionales.

En 1971 el Comité para el Desarrollo Económico (CED, por sus siglas en inglés) se refirió al contrato social entre las empresas y la sociedad, señalando que este se encontraba en profunda transformación. Expresó que la sociedad estaba requiriendo un mayor compromiso y contribución a la calidad de vida y a los valores humanos de parte de los negocios, más allá de su propósito de proveer bienes y servicios (Carroll, 1999). Esta exaltación, explica Carroll (1999), respondió a que en las décadas de 1960 y 1970 los movimientos sociales por la conservación medioambiental, la seguridad del trabajador, los derechos de los consumidores y de los empleados lograron transcender como regulación gubernamental, por lo menos en Estados Unidos. La idea de contrato social adquiere entonces relevancia debido a que los actores sociales cuentan con las herramientas normativas para ejercer presión sobre los intereses de las corporaciones. En este contexto, Yepes et al. (2007) precisan que hacia 1980 se reconoció como fundamental que las organizaciones recuperasen la confianza de la sociedad, introduciéndose en esta línea conceptos como «política pública empresarial, la ética empresarial y la teoría de los stakeholders» (Duque et al., 2013, p. 198), los cuales apuntan al reconocimiento del público y la valoración de la confianza por parte de otros grupos de interés, además de los accionistas.

La década de 1980 y especialmente el final de los noventa transcurrieron en un contexto de globalización de la economía y profundización de la apertura de los mercados, urdiendo preocupaciones respecto a la confianza en las corporaciones, la corrupción y la explotación de los recursos naturales y de la fuerza de trabajo, favoreciendo, a su vez, una sociedad civil que comenzó a fungir también como agente en el transcurso de las definiciones de la RSE (UN Global Compact, 2015). Comenzó a tomarse conciencia de que una población con mayor acceso a la información global, crítica de las prácticas empresariales y en sintonía con preocupaciones ambientales y sociales era capaz de impactar 
las ventas y utilidades de las empresas. Las estrategias filantrópicas, normalizadas para entonces como RSE, comenzaron a resultar insuficientes para satisfacer las demandas de una práctica empresarial ética, ambiental y socialmente comprometida.

En concordancia, los aportes de Wood (1991) y Carroll (1991), respecto al concepto de responsabilidad social de las empresas, incluyen a la sociedad y a la ciudadanía como factores clave en sus desarrollos. De un lado, Wood (como se citó en Duque et al., 2013) propondrá el desempeño social corporativo, definido como «la configuración de principios de responsabilidad social, procesos, políticas, programas y resultados medibles de respuesta social que reflejen la relación de la organización con la sociedad» (p. 198), mientas Carroll (1999) plantea un modelo que sugiere que la responsabilidad social se compone de cuatro tipos: económica, legal, ética y filantrópica, de manera que esta «debe buscar lograr [lucro], obedecer la ley, ser ética, y ser un buen ciudadano corporativo» (p. 289). Ambas definiciones apuntarán, desde distintos ángulos, a la rendición de cuentas a la sociedad.

Durante el siglo XXI se han continuado diversificando las aproximaciones al concepto. Aunque la RSE se ha expandido e institucionalizado en gran cantidad de compañías, autores como Carroll (como se citó en Garriga y Melé, 2004) señalan que en el campo de estudio de la acción empresarial no se cuenta todavía con una definición clara y única. Actualmente, además de los aportes teóricos, pueden rastrearse una serie de iniciativas en materia de política pública y esfuerzos internacionales que responden a la creciente importancia que ha adquirido el debate en torno a la RSE y teorías asociadas, entre las que se destacan la ciudadanía corporativa y la teoría de los grupos de interés (stakeholders), configurando con ello una suerte de marco normativo para delimitar las acciones de RSE, enmarcadas en el clima social del final del milenio (UN Global Compact, 2015). Además, vale la pena mencionar la significativa relación que la RSE ha venido estrechando con el ámbito 
de los derechos humanos ${ }^{1}$ en el entorno empresarial, convirtiéndose estos últimos, si se quiere, en uno de los pilares fundamentales del accionar socialmente responsable de las empresas, sin asumir con ello que la RSE deba circunscribirse únicamente al ámbito de los derechos humanos ni que una política con enfoque de derechos humanos sea equivalente a una de RSE.

Aparecen, en ese sentido, la Global Reporting Initiative (GRI), fundada en 1997 por CERES (Coalition For Environmentally Responsible Economies), el Instituto Tellus y el Programa de las Naciones Unidas para el Medio Ambiente (PNUMA) (Global Reporting Iniciative, s.f.); el Pacto Global, convocado por la Organización de la Naciones Unidas en el Foro Económico Mundial en 1999; y la ISO 26000, que desde 2010 busca, precisamente, "promover un entendimiento común en el campo de la responsabilidad social y complementar otros instrumentos e iniciativas relacionados (...), sin reemplazarlos» (ISO, 2010), en este sentido, define la RSE como:

[La] responsabilidad de una organización ante los impactos que sus decisiones y actividades ocasionan en la sociedad y el medio ambiente, mediante un comportamiento ético y transparente que:

- Contribuya al desarrollo sostenible, incluyendo la salud y el bienestar de la sociedad;

- Tome en consideración las expectativas de sus partes interesadas;

- Cumpla con la legislación aplicable y sea coherente con la normativa internacional de comportamiento; y

- Esté integrada en toda la organización y se lleve a la práctica en sus relaciones.

1 Para citar un ejemplo, además de los derechos humanos como una materia fundamental de la responsabilidad social, la ISO 2600 (2010) menciona: gobernanza de la organización, medio ambiente, prácticas laborales, prácticas justas de operación, asuntos de consumidores y participación activa, y desarrollo de la comunidad. 
Entre tanto, figuran más recientemente los Principios Voluntarios de Seguridad y Derechos Humanos (2000); los Principios Rectores sobre las empresas y los derechos humanos, puesta en práctica del marco de las Naciones Unidas para «proteger, respetar y remediar» (2011) y las Lineas Directrices de la Organización para la Cooperación y el Desarrollo Económicos (OCDE) para Empresas Multinacionales (2011).

Pese a la multiplicidad de desarrollos del concepto, para los fines de esta reflexión, se retomará la definición de RSE construida por Yepes et al. (2007), quienes la sintetizan en siete maneras distintas de entender el concepto:

[La RSE] pretende integrar a la lógica del pensamiento empresarial, que tradicionalmente ha buscado como único objetivo la rentabilidad financiera, los aspectos esenciales del concepto de desarrollo sostenible a través de la promoción y búsqueda del compromiso por parte de las empresas para que en su misma actividad diaria, además de cumplir las leyes, controlen su impacto, como mínimo, en algunos aspectos sociales y ambientales que se pueden agrupar en el respeto a los derechos humanos y la calidad de vida laboral, el compromiso con los valores, la ética empresarial y la transparencia, el cumplimiento de las promesas a clientes, consumidores y socios comerciales, con una operación que impacte cada vez menos al medio ambiente y, de ser posible, apoyando a comunidades vulnerables que estén o no en su área de influencia. (p. 72)

Así definida, se consideran aspectos contemporáneos más prácticos que filosóficos, como lo son: la rentabilidad financiera, el cumplimiento de la legislación, el control del impacto ambiental, la protección a los derechos humanos y derechos laborales, la ética empresarial y la relación con grupos de interés internos (Ej.: socios, clientes y colaboradores) y externos (Ej.: consumidores, comunidades y Gobierno). La responsabilidad social corporativa, entendida bajo estos términos, se orienta a promover el desarrollo sostenible de las comunidades, sin dejar de lado la generación de ganancias y rentabilidad para los accionistas 
de la compañía, pues al encontrarse ligada a las lógicas empresariales esta no deja de considerar la generación de lucro.

\section{El debate voluntariedad-obligatoriedad y las motivaciones para ser socialmente responsable}

Los intentos por ofrecer definiciones del concepto ponen de presente varios puntos de discusión en cuanto a la regulación y la motivación para aplicar políticas de responsabilidad social por parte de las empresas. En este caso, nos referimos particularmente a dos cuestiones que cabe resaltar, en tanto traen a colación reflexiones fundamentales respecto a la operatividad de estas iniciativas hoy en día: la reflexión sobre la voluntariedad u obligatoriedad en las acciones de RSE y las motivaciones para realizarlas.

Sobre el primer punto, existe una postura que defiende el carácter voluntario de las acciones socialmente responsables de las empresas, perspectiva que corresponde de cierta manera con la versión filantrópica de la RSE planteada por Bowen. La voluntariedad implica la toma de acción a partir de la «conciencia de los directivos sobre la influencia que tiene la empresa en su entorno social y por lo tanto, de la ejecución de la actividad productiva de manera responsable y sin presión alguna del gobierno u otras organizaciones» (Yepes et al., 2007, p. 38). Tal como ocurre en el caso Pacto Global —el cual, si bien pretende comprometer a sus países firmantes con la implementación de la sostenibilidad en sus negocios a través de diez principios relacionados con Derechos Humanos, Estándares Laborales, Medio Ambiente, Lucha Contra la Corrupción [y] los Objetivos de Desarrollo Sostenible (ODS) (UN Global Compact, s.f.) - , por su carácter voluntario se trata de iniciativas que, al no ser legalmente vinculantes, no suponen seguimiento estricto o sanciones para las empresas que decidan unirse y no acojan los principios pactados. 
Como contraparte a la voluntariedad, otras posturas afirman que «la responsabilidad social es una obligación de las empresas frente a los actores sociales que se lleva a la práctica a partir del cumplimiento de las normas establecidas sobre las dimensiones ambientales, económicas y sociales» (Yepes et al., 2007, p. 42). Desde esta posición, Jones (como se citó en Yepes et al., 2007) propone que «las empresas tienen obligaciones ante otros grupos de la sociedad, distintos de los accionistas y más allá de los compromisos comprendidos dentro de la ley o de un contrato sindical» (p. 42). En este sentido, la responsabilidad de las empresas superaría a todas luces el mero cumplimiento de la normativa básica para su funcionamiento, pues bajo esta postura, se defiende que una vez suplidas las responsabilidades legalmente establecidas, la empresa debe aceptar y asumir, obligatoriamente, los demás compromisos que surgen del aumento de expectativas por parte de la sociedad.

Actualmente no existe en Colombia legislación alguna que aborde específicamente la regulación de las cuestiones de RSE. Al respecto, Acevedo-Guerrero, Zarate-Rueda y Garzón-Ruiz (2013) señalan que la Sentencia T-247 del 2010 es el primer antecedente jurídico que considera el asunto. En esta sentencia, la Corte Constitucional formula algunas conclusiones que resultan interesantes sobre el tema. Entre ellas, destaca la afirmación de que la RSE

tiene como actores principales a las empresas, pero el compromiso social no debe entenderse agotado en este tipo de programas, que pueden -y deben - ser complementados con la participación de otros actores stakeholders, en terminología de las Naciones Unidas- como la sociedad civilmente organizada, el Estado, los sindicatos, organizaciones con interés social, ONG y organizaciones comunitarias, entre otros. (como se citó en Acevedo-Guerrero, Zarate-Rueda y Garzón-Ruiz, 2013, p. 323)

Así, la Corte puntualizó que las actividades que sean producto de la ejecución de un programa de responsabilidad social no pueden, por esto, desconocer el respeto a los derechos fundamentales y los límites que 
la carta constitucional demarca para sus acciones (Acevedo-Guerrero, Zarate-Rueda y Garzón-Ruiz, 2013).

Entre tanto, algunas iniciativas a tener en cuenta en el caso colombiano en materia de RSE, así como en el ámbito complementario de derechos humanos y empresa — no vinculantes - se hallan en:

- Desde 2003, el Comité Minero Energético de Seguridad y Derechos Humanos, «iniciativa multiactor [que] es una plataforma permanente e independiente de diálogo y construcción colaborativa» (CME, s.f.).

- El Decreto 1400 de 29 de junio del 2012 del Ministerio de Comercio, Industria y Turismo, «por el cual se establece el punto nacional de contacto de Colombia y se adopta el procedimiento previsto en las líneas directrices de la Organización para la Cooperación y el Desarrollo Económicos (OCDE) para empresas multinacionales» (Ministerio de Comercio, Industria y Turismo, 2012, p. 1).

- Las guías de la Fundación Ideas para la Paz, Guía para la debida diligencia en DDHH y DIH en las acciones de fortalecimiento institucional y Guía de mecanismos de quejas y reclamos atentos a los DDHH y al DIH, como «instrumento[s] de autorregulación, de aplicación voluntaria, que busca[n] servir como guía para el desarrollo de la actividad empresarial atenta a los Derechos Humanos y el Derecho Internacional Humanitario» (Fundación Ideas para la Paz, 2014, p. 2).

- La Estrategia Nacional para la Garantía de los Derechos Humanos 20142034, componente de Derechos Humanos y Empresa,

[creada] en el marco del Sistema Nacional de Derechos Humanos y Derecho Internacional Humanitario, [su] propósito [es] incluir, complementar y apropiar, al interior de sus entidades, la «Propuesta de Política Integral de Derechos Humanos 2014-2034», para lo cual se definieron lineamientos por contenidos de derechos, así como estrategias y líneas de acción. (Consejería Presidencial para los Derechos Humanos, 2014, p. 4) 
- El Plan Nacional de Acción sobre Derechos Humanos y Empresas, instrumento de política pública [en el marco del Sistema Nacional de Protección de Derechos Humanos] construido de manera participativa con empresas, organizaciones de la sociedad civil y el apoyo de la Comunidad Internacional, para garantizar el respeto a los derechos humanos en las actividades empresariales. (Consejería Presidencial para los Derechos Humanos, 2016, p. 4)

- La Guía de compras públicas socialmente responsables, «lineamientos [para] las Entidades Estatales y proveedores para el respeto de los derechos humanos y el fomento de las conductas responsables en la compra pública» (Colombia Compra Eficiente, 2018, p. 3).

- La Resolución 40796 del 1 de agosto del 2018 del Ministerio de Minas y Energía, «por medio de la cual se adopta la Política de Derechos Humanos del Sector Minero Energético» (p. 1).

Una vez entendido el alcance de ambas posturas, proponemos que el punto central del debate de voluntariedad-obligatoriedad alrededor de las acciones de RSE recae en que el carácter voluntario de las acciones empresariales y las prácticas éticas que estas subsumen se asocian a la autonomía de la organización y de sus dirigentes. En este sentido, se reconoce que «la empresa adquiere la competencia de realizar acciones éticamente válidas por sí misma, al margen de presiones legales o sociales» (Yepes et al., 2007, p. 41). Se discute entonces, que, al entender las iniciativas desde la voluntariedad, es posible rastrearlas al compromiso de los directivos (voluntad) de actuar guiados por la ética, en lugar de ser persuadidos por presiones externas.

No debería olvidarse que las causas que motivan a las empresas a emprender acciones de responsabilidad social corporativa pueden ser, en efecto, de carácter ético. Por un lado, las cuestiones éticas y morales hacen referencia a los principios establecidos por la compañía. Yepes et al. (2007) señalan que, actualmente, las acciones de responsabilidad social son percibidas como asociadas a la gestión general de la compañía, 
integrándolas como un aspecto prioritario en sus políticas y estrategias. Se destaca así, que las empresas deben tener parámetros éticos de comportamiento. Esta idea, basada en un sentido del bien común, es apoyada por la Conferencia de las Naciones Unidas sobre Comercio y Desarrollo (UNCTAD), quienes plantean que la sociedad otorga a las entidades jurídicas, dentro de las que se cuentan las empresas, una «licencia para operar» que delimita sus derechos y obligaciones, por lo que «el mayor poder de las empresas debe ser compensado por una noción de prácticas comerciales éticas» (Yepes et al., 2007, p. 49).

No obstante, las motivaciones que pueden tener las empresas para la implementación de estrategias de RSE no se agotan exclusivamente en su responsabilidad ética. Al respecto, algunos autores defienden que la finalidad de la responsabilidad social recae en la necesidad de tomar medidas que permitan aumentar la competitividad de la empresa, de tal forma que no se desliga de una visión de negocios. En algunos casos se podría afirmar, en consecuencia, que las acciones socialmente responsables hacen parte de una estrategia diseñada para fortalecer su competitividad, agregar valor a sus procesos y garantizar la estabilidad de sus operaciones a futuro. Para el Banco Interamericano de Desarrollo, esta estrategia redunda en aumentar el valor agregado, mejorando así la competitividad de las empresas (Yepes et al., 2007, p. 56), de manera que, por ejemplo, invertir en campañas de marketing social y realizar programas o donaciones a poblaciones vulnerables contribuyen a hacer más atractiva la imagen de la empresa, visibilizarla en el mercado y mejorar las relaciones con sus clientes y consumidores.

\section{La RSE como herramienta para la gestión de los conflictos}

Dentro de las ciencias sociales, las posturas sobre los conflictos son diversas. Las más tradicionales, como la funcionalista, los consideran como manifestaciones negativas en las que la preocupación por el orden, la uniformidad y el consenso en las sociedades se ven afectadas. Restrepo (2005) señala que el funcionalismo entiende el conflicto como 
una patología del sistema social, como condiciones anormales de las sociedades, es decir, exterioridades a las que se ven sometidos individuos y grupos dentro de un orden social. Sin embargo, para autores como Galtung (1996), los conflictos no pueden tomarse por anomalías, sino que deben ser comprendidos desde sus causas estructurales. En su línea, Restrepo (2005) explica que los conflictos no representan fallas sociales sobre el orden y el consenso, sino que, por el contrario, son parte del orden, no solo como ideas referenciales puestas en contexto, sino también en el sentido según el cual el conflicto en sí mismo tiene una pauta, una lógica que es comprensible y que puede ser observada. Por tanto, los conflictos no pueden pensarse como representaciones caóticas en las sociedades que se oponen a un orden y un consenso universal (como quisieran las posturas funcionalistas). En contraste, deben comprenderse como productos ordenados y en cierta medida, consensuales, que obedecen a las mismas lógicas de las leyes culturales que rigen a las sociedades e individuos.

Siguiendo estas ideas, Marx había planteado ya que precisamente los conflictos entre diferentes grupos y actores sociales constituirían una lucha de clases, el motor de cambio de una sociedad a otra. La importancia del marxismo en los estudios posteriores sobre los conflictos ha sido significativa. Autores como Dahrendorf (1959) retomaron elementos de la teoría marxista como la importancia de la lucha de clases, que vincula estrechamente al conflicto con el cambio social, sugiriendo que en las sociedades modernas los conflictos surgen de la distribución de la autoridad, la participación o no del poder, en tanto que la determinación económica de las clases (lucha social de Marx) resulta solo una manifestación particular del fenómeno de clases. En otras palabras, la determinación económica de las clases y la lucha por una distribución equitativa del factor económico es relevada en las sociedades modernas por la preocupación de la distribución de autoridad, del acceso al poder. Para Touzard (1981), en esta misma línea, el conflicto es una situación definida por las relaciones que se entretejen entre grupos, organizaciones o individuos, donde se persiguen fines contradictorios y 
valores irreconciliables en un contexto de relaciones de poder. Para él, la estructura social de determinada sociedad explica el tipo de conflicto a generarse, por lo que un conflicto parece dar más información - de lo que se creyera- sobre la visión y el tipo de sociedad que lo produce.

Resulta ahora imprescindible reflexionar acerca del tipo de estudios presentados y de sociedades e individuos sobre los cuales son planteadas estas teorías, pues las elaboraciones presentadas hasta el momento tienden a ser propias de sociedades europeas. Según Stavenhagen (1991), en los últimos años ha disminuido el número de conflictos interestatales clásicos y ha aumentado el número de conflictos intraestatales, particularmente en los países que el autor denomina «de Tercer Mundo». Esta «tercermundización» de los conflictos es planteada por Stavenhagen en referencia, fundamentalmente, a los conflictos étnicos que se presentan al interior de los países. Para él, estos conflictos resultan fundamentales para pensar las confrontaciones, pues hacen contrapeso a la universalización de las posturas teóricas europeas que, pese a estudiar la estructura de los conflictos y su importancia contextual, dejan de lado otro tipo de conflictos que no encajan fácilmente en estos modelos analíticos. Como se dijo, las teorías sobre conflicto formuladas en el pensamiento europeo han planteado el cambio social como el resultado de la resolución de los conflictos, un avance entre un estado inicial y uno final, donde lo que está en medio es una etapa conflictiva. Así, la mayoría de las posturas presentadas hasta ahora comprenden el cambio social como el paso de un «algo en conflicto» a una resolución evolutiva que da lugar a un estado mejor del que se partió; un estado aparentemente más «moderno». Planteado de esta manera, los conflictos en países latinoamericanos estarían aún en la "premodernidad» (Stavenhagen, 1991).

Los conflictos de carácter étnico estarían, entonces, por fuera del marco analítico de los estudios tradicionalistas, pues no presentan dentro de su marco de análisis intereses económicos, en los que los actores colectivos tiendan a ser clases sociales definidas en función de su posición 
en el sistema productivo. Cuando las relaciones sociales de producción son esenciales para determinar las relaciones de poder en una sociedad, los problemas étnicos pueden parecer de importancia secundaria. Algunos estudios plantean, de hecho, la relación de la diversidad étnica de los países con su escaso desarrollo económico (Montalvo y ReynalQuerol, 2005; Easterly y Levine, 1997). Dichas perspectivas analíticas que se obstinan en contraponer a los grupos étnicos con el desarrollo han derivado en que los estudios sobre los denominados conflictos étnicos en países de América Latina sean relativamente nuevos.

Para el caso colombiano, es innegable que existen conflictos que se pueden inscribir en las posturas planteadas por Stavenhagen (1991), es decir, aquellos que se escapan al análisis tradicionalista y que no son presentados desde la universalización de conflictos nacionales o internacionales, sino como intraestatales. Estos serían conflictos «a menor escala» que involucran diversos actores, quienes no parecen encerrar en sí mismos clases sociales definidas, y entran en tensión con otros actores sociales o institucionales. En Colombia, estos han sido presentados como conflictos interculturales y conflictos interétnicos. Para Duarte (2015), los conflictos entre actores rurales como lo son los grupos indígenas, afrocolombianos, campesinos y mestizos, tienen una de sus principales causas en la Constitución de 1991 en tanto modelo de gestión del Estado. En ella, si bien se reconoció la diversidad cultural del país, se estableció un esquema diferencial que ha fragmentado y dividido los derechos de las comunidades rurales bajo una perspectiva multiculturalista.

De esta manera, la interculturalidad en el país aparece asociada a un modelo multicultural que entregó valor y reconocimiento a lo étnico, pero de algún modo, introdujo la diversidad desde la diferenciación. Este hecho - unido a una ausencia de ejercicio de ordenamiento territorial, dentro del marco constitucional, que respondiera a la diversidad cultural y étnica del país y sus maneras de aprehender y entender el territorio - ha motivado y agudizado numerosos conflictos por la tierra y 
por la igualdad de derechos entre los actores rurales que conviven en la Nación (Duarte, 2015). Esta situación ha ocasionado diversas tensiones en la relación con estas comunidades por parte del Estado colombiano y su vínculo con otro tipo de instituciones, como ocurre en el caso de las empresas. Los desencuentros que se presentan entre estos actores resultan, como se ha mencionado, de las aspiraciones en común que se tienen sobre algo determinado, usualmente relacionadas con diferentes concepciones de lo que se entiende por desarrollo. Para Duarte (2015), las principales tensiones que surgen entre las diversas aspiraciones de los actores están relacionadas con el territorio, debido, entre otras, a las diferencias que existen

entre las expresiones globales y nacionales del capitalismo rural con relación a los derechos de los pobladores rurales para habitar un espacio que, de manera formal o informal, han construido y territorializado por generaciones. Fenómenos como el despojo territorial, el desplazamiento forzado, el acaparamiento de tierras y la amenaza a la sostenibilidad ambiental de numerosos espacios geográficos del territorio nacional [son consecuencias de esto]. (p.18)

Ahora bien, para Muñoz, Duarte, Rodríguez, Peña, Biel y Salcedo (2014), existen tres causas de emergencia de tensiones y conflictos en relación con la interculturalidad e interetnicidad en el país. En primer lugar, se encuentra la fragmentación de las comunidades por la política diferencial establecida en la Constitución de 1991, la cual, como se mencionó, introduce un esquema diferencial en cuestión de acceso a derechos de las comunidades rurales. En segundo lugar, identifican como causa el mantenimiento de un modelo territorial de gran propiedad y concentración de tierra, que ha profundizado la inequidad de tenencia de la tierra en el país. En tercer lugar, se encuentra el aumento del hacinamiento en áreas de pequeñas propiedades territoriales y, por ende, el aumento de necesidad de tierras de campesinos, indígenas, y afrodescendientes. Sobre estos tres factores comunes se erigen toda serie de contingencias, causalidades y en general, una compleja serie de acontecimientos que 
generan conflictividades entre las comunidades, entre estas con el aparato estatal y con los ejercicios empresariales vecinos.

Desde esta perspectiva y teniendo como referente el trabajo de investigación realizado por el Instituto Estudios Interculturales (IEI) de la Pontificia Universidad Javeriana Cali, se ha identificado que los tres actores susceptibles de entrar en conflicto en la ruralidad colombiana son las comunidades, las empresas y el Estado. Las comunidades son entendidas como las organizaciones y los procesos colectivos de poblaciones indígenas, campesinas y afrodescendientes e incluyen, a su vez, a las organizaciones que los representan, a sus líderes y a sus apuestas programáticas, ideológicas y las procedencias históricas que les dan sentido político en los procesos de relacionamiento con otras comunidades, así como con el Estado y las empresas (IEI y ANT, 2018). Por otra parte, el Estado es concebido a través de dos factores: por un lado, se comprende en su versión más liberal como la institución que emerge de la sociedad misma para regular su funcionamiento, que existe gracias al tributo público y entre cuyas funciones está ser el garante de los derechos de todos los ciudadanos, y por otro lado, el Estado entendido desde sus funcionarios, es decir, desde la complejidad de sujetos que lo componen, sus trayectorias y culturas particulares, así como también los diferentes intereses políticos que ponen en juego. En tercer lugar, las empresas son comprendidas, en primera instancia, como iniciativas privadas cuyo fin es de carácter económico. Sin embargo, con las nuevas reglamentaciones las grandes empresas son fundamentales a la hora de pensar en la sostenibilidad en el territorio y la región, ya que el reto permanente debe ser alcanzar una responsabilidad social y ambiental en sus actividades, coherente con la normativa nacional e internacional, que lleve a una política laboral justa y acorde con los derechos de los trabajadores, así como una explotación responsable de los recursos naturales que no signifique el agotamiento de los mismos (IEI y ANT, 2018).

De este modo, resulta útil traer igualmente a colación la tipología de conflictos territoriales desarrollada por el mismo IEI, en la cual exis- 
ten cinco tipos identificables: 1) conflictos territoriales interculturales; 2) conflictos territoriales ocasionados por la ausencia u omisión estatal; 3) conflictos territoriales por el modelo económico nacional; 4) conflictos territoriales de carácter interno y 5) conflictos por actores armados. De los anteriores y dado el propósito de nuestro artículo, nos centraremos en el tercer tipo, conflictos territoriales por el modelo económico nacional, entendidos como

luchas relativas a los intereses de explotación de la tierra en tanto recurso y las visiones de desarrollo. Por lo general, intervienen como actores empresas (típicamente multinacionales), el Estado, cuando respalda o ejecuta megaproyectos, y las comunidades que tratan de proteger sus territorios, usos y costumbres. Estos conflictos pasan por la inequidad en el acceso a tierras, que se traduce en fallas estructurales para la provisión de derechos, bienes y servicios básicos que aseguren su calidad de vida y satisfacción de necesidades básicas, y se profundizan con las pretensiones de actores armados ilegales sobre el territorio, quienes a través del uso ilegítimo de la fuerza generan desplazamiento y despojo y un control geopolítico sobre el territorio. (IEI y ANT, 2018, p. 7)

Como se mencionó, una de las causas más importantes de los conflictos comunidad-empresa, si no la principal, es que el modelo de desarrollo neoliberal en el cual se enmarca la actividad empresarial no pocas veces se encuentra en contradicción, o por lo menos en debate, con los planes de vida de las comunidades y sus organizaciones sociales ${ }^{2}$. El

2 La resistencia y los debates propuestos por las organizaciones sociales frente al paradigma neoliberal no supone la esencialización de las comunidades rurales y étnicas bajo la identidad del «buen salvaje», que los reivindica como custodios de la riqueza cultural y ecológica. Lo anterior ha sido construido a partir de las definiciones antropológicas que los han relacionado con imaginarios ecológicos y conservacionistas. Por el contrario, el accionar de las comunidades debe complejizarse entendiendo que estas también detentan intereses propios y estratégicos que buscan posicionar en un entramado de relaciones de poder. Sin embargo, dado que no es el objetivo de este artículo, dicha consideración no será ahondada en el texto. 
neoliberalismo, como ha puntualizado Harvey (2007), puede entenderse como:

Una teoría de prácticas político-económicas que afirma que la mejor manera de promover el bienestar del ser humano consiste en no restringir el libre desarrollo de las capacidades y de las libertades empresariales del individuo, dentro de un marco institucional caracterizado por derechos de propiedad privada fuertes, mercados libres y libertad de comercio. (p. 6)

En este sentido, el neoliberalismo también aboga por la apertura de fronteras económicas, que para los países del sur global se traduce, entre muchos otros fenómenos, en la firma de tratados de libre comercio que afectan el consumo de productos locales, y en la concesión de permisos para explorar y explotar recursos naturales del subsuelo por parte de empresas multinacionales, las cuales expanden y profundizan las actividades extractivas en zonas rurales. Muchas veces, las acciones empresariales, ejecutadas en el marco de este modelo y promovidas por las políticas estatales, entran en tensión con los proyectos ambientales, económicos y autonómicos de las comunidades rurales y étnicas.

Este fue el caso, por ejemplo, de la desviación del arroyo Bruno en el municipio de Albania en La Guajira, obra licenciada por la Corporación Autónoma de La Guajira (Corpoguajira) como parte del plan de ampliación de Carbones del Cerrejón Limited para aumentar su explotación de carbón en 3 millones de toneladas anuales (Noguera, 2016). La desviación de 3,6 kilómetros del recorrido de este cuerpo de agua, que sería movido 700 metros hacia el norte, le permitiría a la compañía acceder a una reserva de 35 millones de toneladas del mineral resguardadas bajo su cauce (Silva, 2015). Sin embargo, a finales del 2016, en fallo de segunda instancia, el Consejo de Estado determinó suspender dicha obra, ratificando la sentencia del Tribunal Administrativo de La Guajira proferida en mayo del mismo año en respuesta a la tutela interpuesta por miembros de la comunidad La Horqueta 2 del municipio de Albania. Lorenza Marcela Gil Pushaina, autoridad tradicional de esta comunidad 
constituida por más 80 familias indígenas wayúu, interpuso una acción de tutela en la que argumenta que esta población no fue objeto de Consulta Previa por parte del Cerrejón. La magistrada ponente dra. Veloza Parra, acogiendo lo demandado por Gil Pushaina, expone que el desvío del arroyo afectaría «sus derechos fundamentales a la supervivencia» en términos socioculturales y de acceso al recurso hídrico. Acogiendo lo expuesto por la demandante, el Consejo de Estado en fallo de segunda instancia ordenó a la ANLA, a El Cerrejón y a la Dirección de Consulta Previa del Ministerio del Interior llevar a cabo «un proceso de consulta con la comunidad La Horqueta 2 sobre las formas menos lesivas en que la obra [...] se puede conciliar con las condiciones actuales de vida y los futuros intereses de dicha comunidad» (Corte Constitucional, Auto 419, 2017), el cual sería extensivo a las demás comunidades wayúu en los municipios de Albania o Maicao que resulten directamente afectadas con la modificación del cauce del arroyo.

Este caso permite evidenciar cómo el proyecto expansivo y extractivo de una empresa, por lo demás respaldado por las políticas gubernamentales de desarrollo, puede ir en detrimento de los intereses ambientales y socioculturales de una comunidad étnica, en tanto el acceso al agua en una región donde esta resulta escasa, se relaciona directamente con su consumo, su uso en la agricultura, pesca e incluso, en actividades espirituales propias de su cosmovisión. Adicionalmente, en el caso de poblaciones étnicas como la wayúu, las compañías deben prever dentro de sus proyectos el adecuado cumplimiento de la normatividad vigente que corresponda como lo es el proceso de Consulta Previa, en tanto constituye un derecho fundamental de los sujetos colectivos de protección especial.

A nivel general, la emergencia de conflictos entre comunidad y empresa se ha identificado en dos niveles: uno macro y uno micro (Muñoz et al., 2014). El primero hace referencia a la inscripción de la empresa en las lógicas mercantiles al momento de ser creada, es decir que esta debe regularse bajo las normas internacionales y nacionales a nivel ambiental 
y social, pero, de manera similar, está inscrita en la lógica de lograr el máximo de rentabilidad y la reducción de costos de sus operaciones. En el caso del Cerrejón y el arroyo Bruno, esto se representa en que la necesidad de aumentar su capacidad productiva en la explotación de carbón debe cumplirse atendiendo paralelamente a la normatividad vigente frente a la participación y afectación de los actores sociales en el área de influencia del proyecto. El segundo nivel está directamente relacionado con el primero, y muestra el orden de lo micro, es decir, el relacionamiento entre los integrantes de la misma organización y los representantes de la comunidad local que la conforman. En este último nivel se podrían mencionar los conflictos laborales al interior de la empresa y la garantía de los derechos humanos en el ambiente organizacional. En el microcontexto, se pueden citar, entonces, los conflictos laborales, en el marco de los derechos a la libre asociación y negociación colectiva, ejemplo de ello fueron los surgidos entre los corteros de caña de azúcar y los ingenios azucareros entre los años 2007 y 2008 en el Valle del Cauca. A partir de la experiencia del IEI en la gestión de estas últimas conflictividades, se postula también que dicha tensión «involucra un componente intercultural debido a los choques de las diferentes cosmovisiones entre la lógica empresarial y la cultura de la comunidad afro que integra gran parte de los ingenios azucareros» (Muñoz et al., 2014, p. 15), situación recurrente en numerosos casos de conflictos empresa-comunidades. Como se intuye, en efecto, las tipologías de conflictos antes expuestas no son necesariamente excluyentes entre sí.

De esta manera, vemos cómo, en consecuencia, las acciones enmarcadas en la RSE y en el orden del ámbito complementario de derechos humanos y empresas resultan ser una de las formas en que estas pueden abordar las conflictividades existentes entre las comunidades que se ven impactadas por sus operaciones o bien, entre las comunidades y las mismas empresas, todo ello bajo el entendido de que la gestión oportuna de dichos conflictos, asumiendo que estos son inherentes a las sociedades humanas, pueden generar beneficios de diversa índole 
a la empresa, entre tanto su no atención podría resultar en detrimento de sus intereses. No debería, entonces, resultarnos extraño que, precisamente, entre los factores a tener en cuenta para la evaluación del riesgo que hace parte de los Principios Voluntarios de Seguridad y Derechos Humanos (2000) se haya considerado el análisis de conflictos presentes o futuros en el área de influencia de las empresas. De esta manera, el reto está en que más allá del análisis de riesgo, las empresas asuman un papel activo y sostenible con relación a tales situaciones, apostando a ello desde una perspectiva intercultural.

En el caso particular de las empresas que operan en sectores rurales, lo anterior adquiere especial importancia, puesto que en países como el nuestro es la ruralidad el escenario donde no solo conviven diversidad de actores no menos propensos a entrar en conflicto - tal como ocurre con los conflictos interétnicos e interculturales antes mencionados-, sino que, es además el territorio donde el conflicto armado ha hecho mayor mella. En consecuencia, resulta de vital importancia que las empresas se cuestionen sobre el rol de sus iniciativas de RSE como apuesta para la sostenibilidad de sus negocios, la cual depende en gran medida de su relación con el entorno que les rodea.

Pese a lo anterior, es igualmente innegable que las limitaciones expuestas en torno al debate voluntariedad-obligatoriedad sobre la RSE, así como la ausencia de una base clara - que establezca unívocamente en qué consisten efectivamente las acciones socialmente responsablesgeneren panoramas como el nuestro, en el que se ha llamado «responsabilidad social empresarial» a iniciativas tan disímiles como donaciones únicas, hasta programas de largo aliento con distintas poblaciones. En ese sentido, una propuesta que podríamos formular para lograr una mejor sincronía entre las iniciativas de RSE (y sobre derechos humanos) y la gestión de conflictos con comunidades rurales partiría de la revisión a la apuesta del IEI y la Agencia Nacional de Tierras en su Protocolo para el manejo y tratamiento de conflictos, alertas y estrategias de reacción inmediata (2018), producto de la experiencia conjunta de ambas 
instituciones en relación con diferentes espacios de interlocución entre comunidades rurales y diversos actores a lo largo del territorio colombiano. El Protocolo plantea un paso a paso para la gestión de conflictos inscrito en cuatro momentos: 1) identificación del conflicto; 2) caracterización y contexto del conflicto; 3) construcción de una estrategia de intervención del conflicto y 4) seguimiento de acuerdos y evaluación.

Tomando en cuenta lo anterior, ¿cuál podría ser la relación entre los pasos para la gestión de conflictos y las iniciativas de RSE y derechos humanos, y empresa? La clave está, a nuestro juicio, en utilizar estas iniciativas como herramientas en el proceso de gestión de conflictos, particularmente en el momento de construir una estrategia de intervención del conflicto - cuyo efectivo funcionamiento depende sin duda los otros pasos del proceso-, propiciando con ello ejercicios de incidencia activa en el territorio - más allá de la tan cuestionable neutralidad-y no acciones aisladas que puedan redundar sobre otras ya realizadas o incluso, empeorar las condiciones de las poblaciones involucradas en el conflicto.

Ahora bien, en este contexto es importante detenerse a analizar las condiciones diferenciadas de los actores sociales con que las empresas pueden encontrarse en el territorio rural de nuestro país, a saber: las comunidades indígenas, afrodescendientes y campesinas. Sobre esto último, se hará un énfasis particular en la relación que tiene el debate voluntariedad-obligatoriedad y el relacionamiento con las comunidades especialmente campesinas. Para ello, es imprescindible fijarse en la asimetría del sistema diferencial de derechos - característica del modelo multicultural del Estado colombiano-, derechos que «solamente han funcionado en relación con las minorías autoadscritas a las lógicas multiculturales “indígenas y afrodescendientes”, mientras que los porcentajes mayoritarios de la población, los cuales no se autodefinen con criterios étnicos [entre los que se encuentra la población campesina], son pobremente incluidos en las ventajas y prerrogativas estatales» (Laurent; Zambrano y Chaves, como se citó en Duarte, 2015, p. 28). 
Es, entonces, cuando podríamos cuestionarnos sobre la obligatoriedad que las empresas tienen al establecer relaciones con las comunidades étnicas ubicadas en sus áreas de influencia y en analizar y minimizar dichos impactos, fundamentalmente porque estas obligaciones están ligadas con un ámbito que está efectivamente más allá de la RSE como lo es el derecho vinculante a la Consulta Previa. Esto, en comparación con los motivos, quizá menos apremiantes, que tendrían las empresas para incluir en sus estrategias de relacionamiento e iniciativas de RSE a comunidades campesinas, que no son sujeto de derechos de especial protección como ocurre en el caso de la Consulta Previa de que gozan las étnicas. En consecuencia, ¿son las comunidades campesinas menos merecedoras de ser tenidas en cuenta en iniciativas de RSE? Nuestra respuesta se inclina, sin lugar a duda, hacia una negativa, aduciendo como razón fundamental que precisamente la RSE no se limita — ni regula - por un ámbito normativo de obligatoriedad, de manera que esta tiene la facultad de incluir a la totalidad de los actores presentes en el área de influencia empresarial, sean estos o no sujetos de especial protección ${ }^{3}$, contribuyendo indirectamente con ello a subsanar las asimetrías en cuanto a derechos de unos y otros. Recordemos, entonces, la definición que Yepes et al. (2007) hacen de RSE, elegida para guiar nuestra reflexión, según la cual además de cumplir las leyes, las empresas están llamadas a apoyar a las comunidades vulnerables que estén o no en su área de influencia, de manera que en contextos culturalmente diversos como el nuestro, reconocer tal vulnerabilidad pasa por tomar conciencia sobre el impacto que podría tener la operación empresarial no solo en cuanto generación de conflictos empresa-comunidades, sino también en la generación o profundización de conflictos interétnicos e

3 Vale la pena tener en cuenta que, en el 2018, la Asamblea General de las Naciones Unidas aprobó la Declaración sobre los Derechos de los Campesinos y Otras Personas que Trabajan en las Zonas Rurales, un paso significativo en el reconocimiento del campesinado como sujeto político y de derechos, si se tiene en cuenta además que un camino similar recorrió el reconocimiento que como tal se otorgó desde la década de 1990 a comunidades indígenas y afrodescendientes. 
interculturales al optar por estrategias priorizadas sobre la base de la etnicidad de los actores.

\section{Conclusiones}

Pese a la amplia gama de definiciones en torno a la Responsabilidad Social Empresarial y en ese orden, a la ausencia de una idea unívoca sobre qué es, en efecto, actuar de forma socialmente responsable, así como ante el hoy por hoy irresuelto dilema sobre la voluntariedad u obligatoriedad de la aplicación del concepto, lo cierto pareciera ser que cada vez más las empresas, ya sea por motivaciones de índole ética o económica, tienden a prestar atención a las condiciones en que desarrollan sus actividades y con ellas, a las comunidades a quienes pueden impactar. Así, en casos como el colombiano, marcados por una ruralidad convulsa de conflictos tanto interétnicos como interculturales, acompañados por el recurrente conflicto armado, adquiere sentido pensar la RSE como una herramienta para la gestión de dichos conflictos, toda vez que, de no ser tenidos en cuenta, estos pueden desembocar en detrimento de los intereses de las empresas e incluso limitar sustancialmente sus actividades. Ahora bien, vale la pena tener en cuenta que en el ámbito de la RSE es apremiante considerar la gestión de los conflictos no solo cuando estos involucran directamente a las empresas, sino también cuando estos son del orden intercultural o interétnico, involucrando a las comunidades de sus áreas de influencia, pues se deberá considerar que también estos pueden influir en el logro de sus objetivos.

Siendo así, es fundamental que las empresas no se conviertan en actores que limiten la gestión de conflictos o peor aún, que sean actores partícipes directamente en estos, sino que, por el contrario, asuman un rol activo dentro de su gestión, dado que esto puede impactar significativamente en la sostenibilidad - o no- de sus negocios en territorios tan complejos como el nuestro. Para ello, resulta útil que las empresas no solo comprendan la asimetría de derechos en que se encuentran - y conviven - los actores presentes en los entornos rurales donde 
desempeñan sus acciones (indígenas, afrodescendientes y campesinos), sino que, además, siendo conscientes de tal asimetría, formulen sus estrategias de relacionamiento e iniciativas de RSE en función de atender la misma, procurando la consecución de condiciones más equitativas y no su acentuación.

En ese orden de ideas, partimos de asumir que ser socialmente responsables debería, en la práctica, ir más allá del mero cumplimiento de los asuntos legales - como ocurre erróneamente en algunos casos al confundir la RSE con el cumplimiento de acciones específicas en el marco de derechos de especial protección para comunidades étnicas-, para plantearse, más bien, estrategias que asuman de forma integral la complejidad de los grupos de interés característicos del entorno rural, desde una perspectiva que apunte hacia la sostenibilidad social. En el caso del campesinado, retomando la premisa de la asimetría de derechos operante en nuestro país, es apremiante que se piense en su inclusión dentro de los stakeholders, según sea el caso, de una compañía que opere en el mundo rural. Esto no solo por cuanto constituye en sí mismo un actor primordial en este contexto y porque en muchos casos guarda, además, estrechas relaciones con los actores étnicos, sino porque también, si se quiere atender motivaciones más racionalistas, nos encontramos ante una apuesta por el reconocimiento del campesinado como sujeto político y de derechos que cobra cada vez mayor importancia en el nivel internacional y que bien podría seguir el camino hacia instancias vinculantes como, en efecto, ocurrió en el pasado con las comunidades étnicas. Sin duda, hallamos en las iniciativas de RSE una oportunidad para «constituir o apoyar canales institucionales para el trámite de acuerdos en medio de la diversidad de concepciones, trayectorias y principios de organización del territorio [...]» (Duarte, 2015, p. 18); una apuesta por gestionar los conflictos a través del diálogo intercultural. 


\section{Referencias}

Acevedo-Guerrero, Javier; Zárate-Rueda, Ruth y Garzón-Ruiz, William (2013). Estatus jurídico de la responsabilidad Social Empresarial (RSE) en Colombia. Díkaion, 22(2), 303-332.

Carroll, Archie (1999). Corporate social responsibility: Evolution of a definitional construct of business and society. Business and Society, 38(3), 268-295.

Colombia Compra Eficiente (2018). Guía de compras públicas socialmente responsables. Recuperado de https://bit.ly/2Z7UHB1.

Comisión de las Comunidades Europeas (julio, 2001). Libro Verde. Fomentar un marco europeo para la responsabilidad social de las empresas. Bruselas. Recuperado de https://bit.ly/1yr9h7d.

Comité Minero Energético de Seguridad y Derechos Humanos [CME] (s.f.). Qué es, propósito y visión del CME [portal web] Recuperado de https://bit. ly/33IX7JQ.

Consejería Presidencial para los Derechos Humanos (2014). Estrategia Nacional para la Garantía de los Derechos Humanos 2014-2034, componente de Derechos Humanos y Empresa. Bogotá: Área de Comunicaciones Consejería Presidencial para los Derechos Humanos. Recuperado de https://bit. ly/2ADHEwO.

Consejería Presidencial para los Derechos Humanos (2016). Plan Nacional de Acción sobre Derechos Humanos y Empresa. Bogotá: Área de Comunicaciones Consejería Presidencial para los Derechos Humanos. Recuperado de https://bit.ly/2NdgEKQ.

Corte Constitucional, Sala Plena (9 de agosto del 2017). Auto 419. M. S. Luis Guillermo Guerrero Pérez. Recuperado de https://bit.ly/2THUDXW.

Dahrendorf, Ralf (1959) Class and Class Conflict in Industrial Society. Stanford: Stanford University Press.

Duarte, Carlos (2015). Desencuentros territoriales Tomo I. La emergencia de los conflictos interétnicos e interculturales en el departamento del Cauca. Bogotá: Instituto Colombiano de Antropología e Historia. Recuperado de https://bit.ly/2Z7VWAb.

Duque, Yenny; Cardona, Marleny y Rendón, Jaime (2013). Responsabilidad social empresarial: teorías, índices, estándares y certificaciones. Cuadernos de Administración, 29(50),196-206. 
Easterly, William y Levine, Ross (noviembre, 1997). Africa's Growth Tragedy: Policies and Ethnic Divisions. The Quarterly Journal of Economic, 112(4), 1203-1250. Recuperado de https://doi.org/10.1162/003355300555466.

Fundación Ideas para la Paz [FIP] (2014). Guía para la debida diligencia en DDHH y DIH en las acciones de fortalecimiento institucional. Bogotá: Secretaría Técnica FIP. Recuperado de https://bit.ly/2NbOTEw.

Galtung, Johan (1996). Peace by Peaceful Means: Peace and Conflict, Development and Civilization. Oslo, Noruega: International Peace Research Institute.

Garriga, Elisabet y Melé, Domènéc (agosto, 2004). Corporate Social Responsability Theories: Mapping the Territory. Journal of Business Ethic, 53, 51-71.

Global Reporting Initiative (GRI) (s.f.). GRI's History. Recuperado de https:// bit.ly/249bbEM.

Harvey, David (2005). A Brief History of neoliberalism. En Varela, Ana (trad.). (2007). Breve historia del Neoliberalismo. Recuperado de https://bit. ly/2XUHqwl.

Instituto de Estudios Interculturales y Agencia Nacional de Tierras [ANT] (2018). Protocolo para el manejo y tratamiento de conflictos, alertas y estrategias de reacción inmediata. Recuperado de https://bit.ly/2Z7smyZ.

International Organization for Standardization [ISO] (2010). ISO 26000:2010. Recuperado de https://bit.ly/2uS27ge.

Ministerio de Comercio, Industria y Turismo (29 de junio del 2012). Por el cual se establece el punto nacional de contacto de Colombia y se adopta el procedimiento previsto en las líneas directrices de la Organización para la Cooperación y el Desarrollo Económicos (OCDE) para empresas multinacionales. [Decreto 1400]. Recuperado de https://bit.ly/2MqRquH.

Ministerio de Minas y Energía (1 de agosto de 2018). Por medio de la cual se adopta la Política de Derechos Humanos del Sector Minero Energético. [Resolución 40796]. Recuperado de https://bit.ly/2NvoliP.

Montalvo, Jose y Reynal-Querol, Martha (2005). Ethnic diversity and economic development. Journal of Development Economics. Recuperado de https:// bit.ly/2P0etPm. 
Muñoz, Manuel; Duarte, Carlos; Rodríguez, Tania; Peña, Adelina; Biel, Israel y Salcedo, Leonardo (agosto, 2014). Hacia un Protocolo de Manejo de Conflictos Interculturales [Documento de trabajo]. Recuperado de https://bit. ly/2z9r084.

Murphy, Patrick (1978). An Evolution: Corporate Social Responsiveness. University of Michigan Business Review, 30(6), 19.

Navarro-García, Fernando (2013). Responsabilidad social corporativa: teoría y práctica. México D.F.: Alfaomega.

Noguera, Susana (2 de abril de 2016). Indígenas wayúu se oponen a la desviación del arroyo Bruno. El Espectador. Recuperado de https://bit. ly/1SvN7WF.

Organización de las Naciones Unidas [ONU] (2011). Principios rectores sobre las empresas y los derechos humanos. Nueva York y Ginebra: ONU.

Principios Voluntarios de Seguridad y Derechos Humanos (2000). Disponible en: http://voluntaryprinciples.org/files/ principios_voluntarios_espanol. pdf

Restrepo, Jaime (2005). Elementos para una Teoría del Conflicto. Centro de Estudios de Opinión. 11. Recuperado de https://bit.ly/2TJhpP1.

Silva, Sergio (7 de marzo de 2015). El arroyo que se le atravesó al Cerrejón. El Espectador. Recuperado de https://bit.ly/2YTMwNA.

Stavenhagen, Rodolfo (1991). Los conflictos étnicos y sus repercusiones en la sociedad internacional. Revista internacional de ciencias sociales. Recuperado de https://bit.ly/2Tddwjr.

Touzard, Hubert (1981). La mediación y la solución de los conflictos. Barcelona: Herder.

United Nations Global Compact (2015). IMPACT: Transforming Business, Changing the World - The United Nations Global Compact. DNV GL, United Nations Global Compact. Recuperado de https://bit.ly/2qMpSmM.

United Nations Global Compact (s.f.) ¿Qué es el Pacto Global? Recuperado de https://bit.ly/2OB3J53.

Yepes, Gustavo; Peña, Wilmar y Sánchez, Luis (2007). La Responsabilidad social empresarial. Fundamentos y aplicación a las organizaciones de hoy. Bogotá, Colombia: Universidad Externado de Colombia. 